\title{
Blockchains + Network Privacy $=$ A Nightmare
}

\author{
Aniket Kate \\ Purdue University \\ USA \\ aniket@purdue.edu
}

\begin{abstract}
The hope that cryptography and decentralization together might ensure robust user privacy was among the strongest drivers of the early success of blockchains like Bitcoin. A desire for privacy still permeates the growing blockchain user base today. Nevertheless, due to the inherently public nature of most blockchain ledgers, users' privacy is severely restricted, and de deanonymization attacks are prevalent. Several privacy solutions have been proposed to solve these issues, and a few have been implemented. Nevertheless, some key challenges remain unresolved and a few among those seem to be unsolvable in the information-theoretic and cryptographic sense. In this talk, we discuss privacy challenges, promising solutions, and unresolved privacy issues with blockchains. In particular, we study prominent privacy attacks, analyze the existing privacy solutions, and finally describe interesting unresolved challenges towards publishing and retrieving transactions privately.
\end{abstract}

\section{Author Keywords}

Blockchain; Cryptocurrencies; Privacy; Anonymity; Payment

Channel Networks; Interoperability

\section{BIOGRAPHY}

Prof. Aniket Kate is an Associate Professor in the computer science department at Purdue university. $\mathrm{He}$ is an applied cryptographer and a privacy researcher. His research builds on and expands applied cryptography, distributed computing, and data-driven analysis to solve security/privacy problems in decentralized environments. His current projects focus on communication freedom and distributed ledgers (or blockchains). He is a recipient of the NSF CAREER Award for 2019.

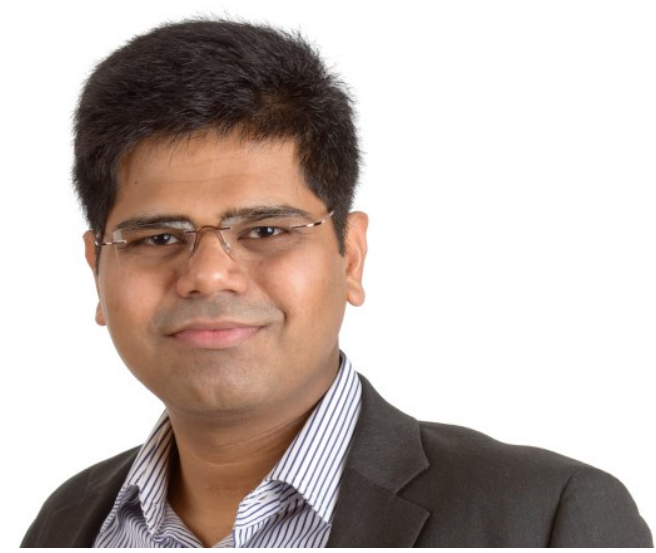

Before joining Purdue in 2015, he was a junior faculty member at Saarland University, Germany. He completed his postdoctoral fellowship at Max Planck Institute for Software Systems (MPISWS), Germany. He has received his $\mathrm{PhD}$ from the University of Waterloo, Canada, and his masters from IIT-Bombay, India.

\section{ACKNOWLEDGMENTS}

Research was sponsored in part by the National Science Foundation (NSF) under grant CNS-1846316 and CNS-1719196, and the IARPA HECTOR program.
Permission to make digital or hard copies of part or all of this work for personal or classroom use is granted without fee provided that copies are not made or distributed for profit or commercial advantage and that copies bear this notice and the full citation on the first page. Copyrights for third-party components of this work must be honored. For all other uses, contact the Owner/Author(s).

$S B C$ '21, June 7, 2021, Virtual Event, Hong Kong.

(C) 2021 Copyright is held by the owner/author(s)

ACM ISBN 978-1-4503-8405-6/21/06

https://doi.org/10.1145/3457977.3460295 